\title{
THERMAL FLUCTUATIONS IN ELECTRIC CIRCUITS AND THE BROWNIAN MOTION
}

\author{
Gabriela Vasziová ${ }^{*}$ - Jana Tóthová ${ }^{*}$ \\ Lukáš Glod $^{* *}$ — Vladimír Lisý ${ }^{*}$
}

\begin{abstract}
In this work we explore the mathematical correspondence between the Langevin equation that describes the motion of a Brownian particle (BP) and the equations for the time evolution of the charge in electric circuits, which are in contact with the thermal bath. The mean quadrate of the fluctuating electric charge in simple circuits and the mean square displacement of the optically trapped BP are governed by the same equations. We solve these equations using an efficient approach that allows us converting the stochastic equations to ordinary differential equations. From the obtained solutions the autocorrelation function of the current and the spectral density of the current fluctuations are found. As distinct from previous works, the inertial and memory effects are taken into account.
\end{abstract}

K e y w or d s: electric circuits, thermal fluctuations, Brownian motion, optical trap, generalized Langevin equation

\section{INTRODUCTION}

The mathematical correspondence between mechanical and electrical properties is often used to construct an electrical model of a given mechanical system [1]. This is a very useful way to predict the performance of a mechanical system, since the electrical elements are inexpensive and the measurements are usually very accurate. Such analog computation has been recently used also for stochastic systems in connection with the applicability of thermodynamic laws on nanoscales $[2,3]$ and with the so called fluctuation theorems that in the last decade attract great attention not only in the statistical and condensed matter physics but also in the very different fields of science from nanotechnology to biology [4-7]. In electric circuits, the fluctuations have long been considered a nuisance - already the seminal works by Johnson and Nyquist on noise caused by thermal agitation of charge carriers were inspired by the problem of noise in telephone wires $[8,9]$. On the other hand, when the studied system produces a frequency dependent (colored) noise, such noise contains information on the system. The information on the properties of the system is obtained from the measurements of the spectral density of the fluctuations, usually those of the current. The analogy with the noisy oscillator or the Brownian motion (BM) of particles can be very useful in the calculations of these fluctuations and interpretation of the measurements in circuits, and vice versa.

In the present work we explore the analogy between the motion of a Brownian particle (BP) dragged by a moving harmonic potential and simple electric circuits in contact with the thermal bath, described by exactly the same equations. Using the methods of statistical physics we calculate the mean square displacement (MSD) of the BP. To do this, we use the approach [10] that allows one to convert the stochastic equations of the Langevin type to ordinary differential equations, which are much easier to solve. To our knowledge, the used method has not been applied to these problems so far. Its efficiency is seen especially in the context of solving the generalized Langevin equations (LEs) that are often used to describe various problems of anomalous BM [11]. Having found the MSD of the BP (which in electric circuits corresponds to the mean quadrate of the electric charge), it is then easy to evaluate the BP velocity autocorrelation function (VAF) (corresponding to the autocorrelation function for the electric current). From these functions we calculate the spectral density of the fluctuations, $e g$, the spectrum of the colored noise produced by the circuits.

In the mentioned attempts to validate the fluctuation theorems $[5,6,12]$ the inertia-less LE [13], applicable only for long observation times, has been solved. Although in the studied systems the inertia time-scale was smaller than the experimental sampling rate so that the simplified LE could be used, for the description of more accurate experiments, see $e g$ [14], this approach is not appropriate. If such a theory is applied to electric circuits [5], it means that they do not include the inductor. Moreover, it is insufficient to generalize the theory simply by the consideration of nonzero mass $m$ of the particle or the inductance $L$ of the inductor. Both in the movement of a colloidal particle in a solvent or in the case of nanoscale circuits in contact with the thermal bath the memory effects should

\footnotetext{
* Department of Physics, Faculty of Electrotechnics and Informatics, Technical University of Košice, Park Komenského 2, 04200 Košice, Slovakia, gabriela.vasziova@tuke.sk, jana.tothova@tuke.sk, vladimir.lisy@tuke.sk; ${ }^{* *}$ Department of Mathematics and Physics, Institute of Humanitarian and Technological Sciences, The University of Security Management, Kukučínova 17, 04001 Košice, Slovakia, lukas.glod@vsbm.sk
} 
be taken into account. As a result, the ordinary LE for the particle position or for the temporal evolution of the charge in a circuit becomes inapplicable [13]. It changes to a Volterra-type integro-differential equation with the stochastic force represented by a colored noise, as distinct from the white-noise force in the standard LE [3]. Our approach to the solution of such generalized LE is exact in the classical approximation and equally applicable to electric circuits and the BM with memory.

\section{BROWNIAN PARTICLE DRAGGED BY OPTICAL TWEEZER}

Let us consider a BP dragged through a solvent by an optical tweezer. According to the experiment [6], the $\mathrm{BP}$ is subject to an external harmonic potential with a time dependent position $x_{t}^{*}$ of its minimum. For $t \leq 0$ this minimum is at the origin, $x_{t}^{*}=0$, whereas for $t>0$ it moves with a constant velocity $v^{*}$. Its motion can be described by the LE [13]

$$
m \frac{\mathrm{d}^{2} x_{t}}{\mathrm{~d} t^{2}}=-\alpha \frac{\mathrm{d} x_{t}}{\mathrm{~d} t}+\xi-k\left(x_{t}-v^{*} t\right)
$$

where $m$ is the particle mass, $x_{t}$ its position at the time $t, \alpha$ is the friction coefficient, and $\xi$ is the thermal white noise with zero mean and the property $\left\langle\xi(t) \xi\left(t^{\prime}\right)\right\rangle=$ $2 k_{B} T \alpha \delta\left(t-t^{\prime}\right)$. Here, $k_{B}$ is the Boltzmann's constant, $T$ is the temperature, $\delta(t)$ is the Dirac delta function and the brackets $\langle\ldots\rangle$ denote the statistical averaging. Equation (1) in its overdamped limit, which corresponds to $m=0$, was used by Mazonka and Jarzynski [15] as an exactly solvable model to illustrate some statisticalmechanical predictions for systems evolving far from equilibrium, and by Van Zon and Cohen [16] to describe the discussed experiment [6]. As distinct from those works, we will proceed with the full form of (1), keeping $m \neq 0$. Due to the motion of the trap the mean value of the particle position, $\left\langle x_{t}\right\rangle \equiv x^{*}$, will be nonzero. We thus divide the searched solution $x_{t}$ in two parts: $x_{t}=x+x^{*}$, where $x^{*}$ obeys the deterministic equation, ie (1) without the random force,

$$
m \ddot{x}^{*}=-\alpha \dot{x}^{*}-k\left(x^{*}-v^{*} t\right),
$$

and for $x$ we have

$$
m \ddot{x}=-\alpha \dot{x}+\xi-k x .
$$

For the MSD $X(t)=\left\langle[x(t)-x(0)]^{2}\right\rangle$ of the BP with respect to the frame in which the motion of the particle is governed by (2) we need the solution of the following equation $[10]$

$$
m \ddot{X}+\alpha \dot{X}+k X=2 k_{B} T .
$$

The initial conditions are $X(0)=V(0)=0$, where $V(t)=\mathrm{d} X(t) / \mathrm{d} t=2 D(t)$. The function $D(t)$ is called the time-dependent diffusion coefficient, which is through the relation $\Phi(t)=\mathrm{d} D(t) / \mathrm{d} t$ connected to the VAF $\Phi(t)=\langle v(t) v(0)\rangle$ [17]. Equation (2) has to be solved with the conditions $x^{*}=\mathrm{d} x^{*} / \mathrm{d} t=0$ at $t=0$. Now the problem is fully formulated. It can be added that if one is interested in the spectral density of fluctuations, then, according to the Wiener- Khinchin theorem, it equals to the Fourier transform of the autocorrelation function of the quantity of interest [18]. So, for the fluctuation spectrum of the velocity we have

$$
S_{v}(\omega)=\frac{2}{\pi} \int_{0}^{\infty} \mathrm{d} t\langle v(0) v(t)\rangle \cos \omega t
$$

One of our main results consists already in the conversion of the stochastic (3) to a simple ordinary differential equation (4). The rule on which this transformation is based on has been long ago formulated by Vladimirsky [10] and states that the MSD $X(t)$ of a BP obeys the same equation as that for the particle position $x(t)$, if the driving stochastic force is replaced by the constant $2 k_{B} T$. In our case (4) can be easily derived from (3) for a stationary process $x$. However, the method has a much broader applicability: it can be used for any stochastic linear equations of motion, including those that describe non-Markovian processes.

Equation (4) has the solution

$$
X(t)=\frac{2 k_{B} T}{k}\left\{1-\frac{\varepsilon_{2} \exp \left(\varepsilon_{1} t\right)-\varepsilon_{1} \exp \left(\varepsilon_{2} t\right)}{\varepsilon_{2}-\varepsilon_{1}}\right\},
$$

where $\varepsilon_{1,2}=-(\alpha / 2 m) \times\left[1 \mp\left(1-4 k m / \alpha^{2}\right)^{1 / 2}\right]$ are the roots of the equation $m \varepsilon^{2}+\alpha \varepsilon+k=0$ (which arise after the substitution $X \sim \exp (\varepsilon t)$ in (4)). Solving the deterministic (2) we obtain

$$
\begin{gathered}
x^{*}(t)=A^{*} \exp \left(\varepsilon_{1} t\right)+B^{*} \exp \left(\varepsilon_{2} t\right)+v^{*} t-\alpha v^{*} / k, \\
A^{*}=v^{*} \frac{1+\alpha \varepsilon_{2} / k}{\varepsilon_{2}-\varepsilon_{1}}, B^{*}=-v^{*} \frac{1+\alpha \varepsilon_{1} / k}{\varepsilon_{2}-\varepsilon_{1}} .
\end{gathered}
$$

When the particle displacement oscillates in the harmonic well, which happens if $1-4 k m / \alpha^{2}<0$, we have $\varepsilon_{1,2}=$ $-\alpha / 2 m \pm i \omega_{0}$, where $\omega_{0}=\left[k / m-(\alpha / 2 m)^{2}\right]^{1 / 2}$,

$$
\frac{X(t)}{2 k_{B} T / k}=1-\exp \left(-\frac{\alpha t}{2 m}\right)\left(\cos \omega_{0} t+\frac{\alpha}{2 m \omega_{0}} \sin \omega_{0} t\right),
$$

whereas the deterministic Eq. (2) has the solution

$$
\begin{aligned}
& x^{*}(t)=v^{*}\left(t-\frac{\alpha}{k}\right)- \\
& \frac{v^{*}}{\omega_{0}} \exp \left(-\frac{\alpha}{2 m} t\right)\left[\left(1-\frac{\alpha^{2}}{2 m k}\right) \sin \omega_{0} t-\frac{\alpha \omega_{0}}{k} \cos \omega_{o} t\right] .
\end{aligned}
$$

In the discussed works $[15,16]$, a much simpler task was considered. The solutions for this case follow from (8) and (9) when $m \rightarrow 0$,

$$
\begin{aligned}
& X(t) \approx \frac{2 k_{B} T}{k}\left[1-\exp \left(-\frac{k}{\alpha} t\right)\right], \\
& x^{*}(t) \approx v^{*}\left[t-\frac{\alpha}{k}+\frac{\alpha}{k} \exp \left(-\frac{k}{\alpha} t\right)\right] .
\end{aligned}
$$


However, the correct approach requires the inertial effects, which are important at short times, to take into account. The behavior of the solution (8) at short times differs from that of (10). In particular, one of the initial conditions for $X(t)$ now cannot be satisfied since the simplified (4) with $m=0$ determines the value $\dot{X}(0)=2 k_{B} T / \alpha$ instead of 0 . We suppose that it would be important to prove the validity of the fluctuation theorems for all the times, not only for long times as in the cited works.

The total MSD of the dragged BP in our case is

$$
X_{t}(t)=\left\langle\left[x_{t}(t)-x_{t}(0)\right]^{2}\right\rangle=\left[x^{*}(t)\right]^{2}+X(t) .
$$

Using it, other correlation functions for the BP, shown after (2), can be calculated. For example, the full VAF, $\left\langle v_{t}(t) v_{t}(0)\right\rangle$, is determined only by the stochastic part (8), $\Phi(t)=\ddot{X}(t) / 2$. It is seen by considering the autocorrelation function for the coordinate $\dot{x}_{t}=\dot{x}+\dot{x}^{*}$ with respect to the laboratory system and taking into account the initial conditions for $\dot{x}^{*}$. Using Eq. (8), we find

$$
S_{v}(\omega)=\frac{1}{\pi} \int_{0}^{\infty} \ddot{X} \cos (\omega t) \mathrm{d} t=-\frac{\omega^{2}}{\pi} \int_{0}^{\infty} X \cos (\omega t) \mathrm{d} t
$$

From the solution (6) we then have

$$
S_{v}(\omega)=\frac{8 k_{B} T}{\pi \alpha}\left(\frac{\alpha}{2 m}\right)^{2} \frac{\omega^{2}}{\left(\varepsilon_{1}^{2}+\omega^{2}\right)\left(\varepsilon_{2}^{2}+\omega^{2}\right)}
$$

or, expressed explicitly through the system parameters,

$$
S_{v}(\omega)=\frac{1}{\pi} \frac{2 k_{B} T \alpha \omega^{2}}{k^{2}+\left(\alpha^{2}-2 k m\right) \omega^{2}+m^{2} \omega^{4}} .
$$

It follows from here that in the limit $m \rightarrow 0$

$$
S_{v}(\omega)=\frac{2 k_{B} T}{\pi} \alpha \omega^{2}\left[k^{2}+(\alpha \omega)^{2}\right]^{-1}
$$

This formula, however, significantly differs from the result that is obtained after the direct use of (10) for $X(t)$ obtained at $m=0$,

$$
S_{v}(\omega)=-\frac{2 k_{B} T}{\pi} \frac{k^{2}}{\alpha}\left[(k)^{2}+(\alpha \omega)^{2}\right]^{-1} .
$$

This again puts in doubt the proof of the fluctuation theorems given in [16].

\section{FLUCTUATIONS IN SIMPLE ELECTRIC CIRCUITS}

The presented theory thus describes, in a more consistent way than in the original works $[6,15,16]$, the stochastic motion of a mesoscale particle dragged through a solvent by a moving harmonic well. The theory is however equally applicable to the description of quite different systems, namely, the electric circuits. Similar works can be already found in the literature, eg $[5,19]$. Equation (1) exactly corresponds to a simple electric circuit in which the resistor with the resistance $R$, the capacitor with the capacitance $C$, and the inductor with the inductance $L$ are connected in series. They are subject to a voltage source $V(t)$. Let the imposed voltage linearly increases with the time $t, V(t)=\kappa t$, and the fluctuations of the voltage drop across the resistor are described by $\delta V(t)$. The equation for such a circuit is

$$
L \ddot{Q}+R \dot{Q}+\frac{1}{C} Q=\kappa t+\delta V(t)
$$

The correspondence between Eqs. (1) and (18) is seen if the particle displacement $x_{t}$ is replaced by the charge $Q, L$ replaces the particle mass $m, R$ and $1 / C$ are for the friction coefficient and the elastic constant $k$, respectively, and the velocity of the harmonic well is changed with the constant $C \kappa$. In another example a resistor and a capacitor are arranged in parallel and are subject to a constant, non-fluctuating current source $I$. Energy is being dissipated in the resistor. The fluctuations that accompany this dissipation are described by a random noise term $\delta V$. The difference from (18) is only in the term $\kappa t$, which is now replaced by $I t / C$. Thus, the current $I$ corresponds to the velocity $v^{*}$. Such electrical systems are of particular interest, because all parameters in the setup can easily be controlled and the measurements are very accurate. Moreover, as distinct from the experiments on real particles, the "mass" (the inductance $L$ ) can be zero so that the corresponding model is easily tractable. All the formulas obtained in this paper are applicable to the above described simple circuits and could be experimentally tested. Particularly it concerns the spectral density of the electric current fluctuations, which are usually observed in experiments. To use our formulas to interpret such experiment, one should just replace in (5) and (13)(17) the particle velocity $v(t)$ with the electric current $I(t)$.

\section{BROWNIAN MOTION AND NANOSCALE CIRCUITS WITH MEMORY}

The traditional LE approach is valid for the long-time motion of BPs in fluids, as in the experiment [6]. It is appropriate also at short times but when simultaneously the density of the particles is much larger than the density of the surroundings; for details see $[14,20]$. A more correct approach requires replacing the Stokes friction force (valid only for the steady motion of particles) by a force that describes also the transition of the particle to the steady state. For incompressible fluids it is the Boussinesq force that takes into account the memory effects in the particle motion. It is natural to expect that the effects of memory exist also in electric circuits. Then the question arises how these effects (ie, an "anomalous" relaxation of the charge or current, which differs from 
the normal behavior of the $\mathrm{BP}$ within the Langevin theory) can be theoretically described. It could be done phenomenologically (modeling the resistivity) or from first principles, explicitly describing the thermal bath, which is the method suitable also for the description of dissipative quantum- mechanical situations. Recently, such a theory has been developed for nanoscale $R L C$ circuits in contact with the thermal bath [3]. The bath was modeled by harmonic oscillators (linear $L C$ circuits) attached to the studied circuit. After the standard canonical quantization scheme applied to the whole closed Hamiltonian system and next tracing out the bath, since only the degrees of freedom of the initial circuit are considered to be observable, the final generalized LE for the charge $Q(t)$ can be obtained. Let the resistor and the capacitor have the resistance $R$ and the capacitance $C, \Gamma$ is the maximal frequency of the bath and $\eta(t)$ is the noise, which, in the classical limit considered here, has the property $\langle\eta(t) \eta(0)\rangle=k_{B} T R \Gamma \exp (-\Gamma t)$. Then the equation for $Q(t)$ is [3]

$L \ddot{Q}+\left(R \Gamma+\frac{1}{C}\right)-R \Gamma^{2} \int_{0}^{t} \mathrm{~d} \tau \exp [-\Gamma(t-\tau)] Q(\tau)=\eta(t)$.

This equation can be solved for the quantity $v(t)=$ $\mathrm{d} \xi(t) / \mathrm{d} t=(\mathrm{d} / \mathrm{d} t)\left\langle[Q(t)-Q(0)]^{2}\right\rangle$, using the already applied rule [10]. We just have to replace in Eq. (19) $Q(t)$ with $\xi(t)=\int_{0}^{t} v(\tau) \mathrm{d} \tau$, and the force $\eta(t)$ with $2 k_{B} T$. The new integro-differential equation is deterministic and can be solved using the Laplace transformation. For the Laplace transformed quantity $\tilde{v}(s)=\mathcal{L}\{v(t)\}$, taking into account the conditions $\xi(0)=v(0)=0$ and applying the convolution theorem, we obtain the following equation

$$
\begin{array}{r}
\tilde{v}(s)=2 k_{B} T \frac{\Gamma+s}{L s^{3}+\Gamma L s^{2}+s(\Gamma R+1 / C)+\Gamma / C}= \\
2 k_{B} T \frac{\Gamma+s}{\left(s-s_{1}\right)\left(s-s_{2}\right)\left(s-s_{3}\right)}
\end{array}
$$

Its solution is straightforward. The inverse Laplace transform can be obtained from this expression after its decomposition to simple fractions $\sim 1 /\left(s-s_{i}\right)$, where $s_{i}$ are the roots of the cubic equation $L s^{3}+\Gamma L s^{2}+s(\Gamma R+1 / C)+$ $\Gamma / C=0$. Then, $e g$, if the roots $s_{i}$ are different, $v(t)$ will be a sum of exponentials $v(t)=\sum_{i=1}^{3} A_{i} \exp \left(s_{i} t\right)$. It is seen from the decomposition

$$
\frac{\Gamma+s}{\left(s-s_{1}\right)\left(s-s_{2}\right)\left(s-s_{3}\right)}=\frac{A_{1}}{s-s_{1}}+\frac{A_{2}}{s-s_{2}}+\frac{A_{3}}{s-s_{3}},
$$

where the constant $A_{1}=\left(\Gamma+s_{1}\right) /\left[\left(s_{1}-s_{2}\right)\left(s_{1}-s_{3}\right)\right]$ is obtained after multiplying this equation by $\left(s-s_{1}\right)$ and then setting $s=s_{1}$; other constants are of the same form with the cyclic change of the indexes $1 \rightarrow$ $2 \rightarrow 3 \rightarrow 1$. The limits of long and short times are determined by the limiting behavior of $\tilde{v}(s)$ at $s \rightarrow 0$ and $s \rightarrow \infty$, respectively. In this way we find $v(t) \approx$ $\left(2 k_{B} T / \Gamma L\right)[1-\exp (-\Gamma t)] \approx 2 k_{B} T t / L$, as $t \rightarrow 0$. This corresponds to $\xi(t) \sim t^{2}$, exactly as in the case of the MSD for the BPs. The autocorrelation function for the current, $i(t)=\langle I(t) I(0)\rangle$, exponentially approaches the constant $k_{B} T / L$, as $t \rightarrow 0$. At long times $v(t)$ and $i(t)$ converge to zero with the relaxation time $R C+1 / \Gamma$, $v(t) \approx 2 k_{B} T C(R C+1 / \Gamma)^{-1} \exp [-t /(R C+1 / \Gamma)]$. The "MSD" $\xi(t)$ at long times approaches the value $2 k_{B} T C$. If $L=0$ from the beginning, the condition $\xi(0)=0$ cannot be satisfied, since the equation of motion would yield $2 k_{B} T=0$. If $L \neq 0$, at $t=0$ we have $L \ddot{\xi}(0)=2 k_{B} T$, so that the expansion at small $t$ is $\xi(t)=k_{B} T t^{2} / L+\ldots$

\section{CONCLUSION}

In conclusion, we have theoretically described the chaotic motion of a colloidal particle optically trapped in a harmonic well that uniformly moves in a solvent. This situation was realized in several experiments but, in our opinion, the original description of them, as an overdamped motion of a Brownian particle, is not satisfactory. Whereas it is appropriate asymptotically for long times, it gives incorrect dependencies of the relevant time correlation functions at short times. Accordingly, their spectral densities show an improper dependence on the frequency. This could be verified in accurate electric measurements on simple circuits in contact with the thermal bath, which are within the approach based on the traditional Langevin equation exact analogues to the Brownian motion. However, the applicability of the Langevin equation to the Brownian motion in suspensions has a strong limitation. When the characteristic times in experiments are comparable to or smaller than $R^{2} \rho / \eta$, where $R$ is the particle radius and $\rho$ and $\eta$ are the density and viscosity of the solvent, the viscous aftereffect should be taken into account. For such time scales the presented solution becomes inapplicable. A similar situation takes place for nanoscale electric circuits, which are now possible to realize experimentally. In both cases the evolution of the studied systems is characterized by some kind of memory. The corresponding Langevin equations thus turn to stochastic integro-differential equations and involved calculations are needed to solve them. Our work represents a progress with such problems. It consists in converting the complicated stochastic equations of motion into the deterministic ones that are quite easily solvable analytically. We have shown that within the classical consideration the exact solution of the generalized Langevin equation can be found. The method is directly applicable to the description of the Brownian motion driven by a colored noise since for the usual micron-sized particles the classical approach is relevant. However, for nanoscale circuits and low temperatures the quantum effects become important $[2,3]$. The future work thus requires a generalization of the presented method of solution to the case of quantum noise. 


\section{Acknowledgement}

This work was supported by the Agency for the Structural Funds of the EU within the projects NFP 26220120021 and 26220120033 , and by the grant VEGA $1 / 0300 / 09$.

\section{REFERENCES}

[1] TANG, K. T.: Vector Analysis, Ordinary Differential Equations and Laplace Transforms. Mathematical Methods for Engineers and Scientists, Part II, Springer-Verlag, Berlin, Heidelberg, 2007.

[2] NIEUWENHUIZEN, T. M.-ALlAHVERDYAN, A. E.: Statistical Thermodynamics of Quantum Brownian Motion: Construction of Perpetuum Mobile of the Second Kind, Phys. Rev. E 66 (2002), 036102.

[3] Allahverdyan, A. E.-NIEUWEnhuiZEN, T. M.: On Testing the Violation of the Clausius Inequality in Nanoscale Electric Circuits, arXiv:cond-mat/0205156v1, Phys. Rev. B 66 (2002), 115309, (see also comment on this article in: Gyftopoulos, E.P. - von Spakovsky, M.R.: Comments on Testing the Violation of the Clausius Inequality in Nanoscale Electric Circuits, arXiv 0706.2842v1 [quant-ph]).

[4] EVANS, D. J.-SEARLESD. J.: The Fluctuation Theorem, Adv. Phys. 51 (2002), 1529-1585.

[5] VAN ZON, R.-CILIBERTO, S.-COHEN, E. G. D. : Power and Heat Fluctuation Theorems for Electric Circuits, arXiv:cond-mat/0311629v2, Phys. Rev. Lett. 92 (2004), 130601.

[6] WANG, G. M.-SEVICK, E. M.-MITTAG, E.-SEARLES, D. J.-EVANS, D. J. : Experimental Demonstration of Violations of the Second Law of Thermodynamics for Small Systems and Short Time Scales, Phys. Rev. Lett. 89 (2002), 050601.

[7] TANiguCHI, T.-COHEN, E. G. D. : Nonequilibrium Steady State Thermodynamics and Fluctuations for Stochastic Systems, J. Stat. Phys. 130 (2008), 633-677.

[8] JOHNSON, J. B.: Thermal Agitation of Electricity in Conductors, Nature 119 (1927), 50-51, Phys. Rev. 32 (1928) 97-109.

[9] NYQUIST, H.: Thermal Agitation of Electric Charge in Conductors, Phys. Rev. 29 (1927), 614, Phys. Rev. 32 (1928) $110-113$.

[10] VLADIMIRSKY, V. V.: To the Question of the Evaluation of Mean Products of Two Quantities Related to Different Moments of Time in Statistical Mechanics, Zhur. Eksper. Teor. Fiz. 12 (1942), 199-202. (in Russian)

[11] KLAGES, R.-GÜNTER, R.-SOKOLOV, I. M., Eds. : Anomalous Transport, Wiley - VCH, Berlin, 2008.

[12] CARBERRY, D. M.-REID, J. C.-WANG, G. M.-SEVICK, E. M.-SEARLES, D. J.-EVANS, D. J.: Fluctuations and Irreversibility: an Experimental Demonstration of a Second Law-Like Theorem using a Colloidal Particle Held in an Optical Trap, Phys. Rev. Lett. 92 (2004), 140601.

[13] COFFEY, W. T.-KALMYKOV, Y. P.-WALDRON, J. T. : The Langevin Equation. with Applications to Stochastic Problems in Physics, Chemistry and Electrical Engineering, World Scientific, New Jersey e.a., $2^{n d}$ Ed., 2005.

[14] TOTHOVA, J.-BRUTOVSKY, B.-LISY, V.: Effects of Hydrodynamic Memory in the Dynamic Light Scattering from Polymer Solutions, Laser Physics 14 (2004), 1511-1515.

[15] MAZONKA, O.-JARZYNSKI, C.: Exactly Solvable Model Illustrating Far-From-Equilibrium Predictions, arXiv:cond-mat/991212v1.
[16] VAN ZON, R.-COHEN, E. G. D. : Stationary and Transient Work-fluctuation Theorems for a Dragged Brownian Particle, Phys. Rev. E 67 (2003), 046102.

[17] MAZO, R. M.: Brownian Motion. Fluctuations, Dynamics, and Applications, Oxford University Press, New York, 2009.

[18] VAN KAMPEN, N. G.: Fluctuations in Nonlinear Systems of Fluctuation Phenomena in Solids, Chapter 5, pp. 139-177, Edited by BURGESS, R. E., Academic Press, New York, 1965.

[19] GARNIER, N.-CILIBERTO, S.: Nonequilibrium Fluctuations in a Resistor, Phys. Rev. E 71 (2005), 060101(R).

[20] LISY, V.-TOTHOVA, J.: arXiv:cond-mat/0410222.

Received 30 June 2010

Gabriela Vasziová was born in Královský Chlmec, Slovak Republic, in 1986. She graduated at the Faculty of Science, Pavol Jozef S̆afárik University in Košice, Slovakia, in Mathematics and Physics (2009). At present she is a PhD student at the Department of Physics, Faculty of Electrical Engineering and Informatics, Technical University in Košice.

Jana Tóthová was born in Prešov, Slovakia. She graduated at the Department of Biophysics, Faculty of Science of the P.J. Šafárik University in Košice, where she was employed as the scientific worker until 2008. Currently she is with the Department of Physics at the Faculty of Electrical Engineering and Informatics, Technical University of Košice. In 2005 she defended the PhD thesis "The Dynamics of Polymers in Solution with Hydrodynamic Memory". Her scientific interests are mainly in the field of spectroscopic and thermodynamic properties of biological macromolecules, their interaction with different agents, and the rheology of polymer solutions.

Lukáš Glod was born in Prešov, Slovak Republic, 1984. He received the MS degree in Mathematics and Physics from University of Prešov in Prešov in 2007. Since 2008 he is a PhD student in General Physics and Mathematical Physics at the Faculty of Science, Pavol Jozef Šafárik University in Košice, Slovakia. His main fields of interest include macromolecular physics, statistical and nonlinear physics.

Vladimír Lisý was born in Moscow, 1953. He graduated in 1977 at the Faculty of Physics, Odessa State University, USSR. From 1977 to 2007 he worked at the P. J. Šafárik University in Košice and then at the Faculty of Electrical Engineering and Informatics, Technical University of Košice. In the period 1982-1990 he was a researcher at the Bogoliubov Laboratory of Theoretical Physics, JINR, Dubna, Russia, where he received his $\mathrm{PhD}$ in theoretical physics. In 1993 he became associate professor of biophysics at the Comenius University, Bratislava, where he also defended the DSc title in chemical physics (2008). From 2009 he is professor in the field of condensed matter physics and acoustics. His main interests are in the soft condensed matter theory.

$* * *$

This work was presented at 16-th International Conference Applied Physics of Condensed Matter, June 16-18, 2010, Malá Lučivná, Slovakia. 